\title{
Giardino Garibaldi in Palermo
}

\author{
Hilke Steinecke \& Peter Schubert
}

\begin{abstract}
The Garibaldi garden is a small municipal park in the historical center of Palermo/Sicily. It is characterised by its old trees. The most impressive trees are three Ficus macrophylla with many thick aerial roots. Together with the large ficus in the botanical garden of Palermo they are the largest ficus trees in Europe.
\end{abstract}

\section{Zusammenfassung}

Der Garibaldi-Garten ist ein kleiner Stadtpark in der Altstadt von Palermo/Sizilien. Der Park weist einen alten Baumbestand auf. Am beeindruckendsten sind drei riesige Feigenbäume (Ficus macrophylla) mit zahlreichen, dicken Luftwurzeln. Einer von ihnen gehört zusammen mit dem gewaltigen Feigenbaum im Botanischen Garten von Palermo zu den größten Ficus-Bäumen in Europa.

\section{Ruhe inmitten der Großstadt}

Palermo zieht jährlich zahlreiche Touristen an, die vor allem die großartigen Baudenkmäler Dom und Normannenpalast aufsuchen. In der hektischen Atmosphäre dieser verkehrsreichen Großstadt Siziliens findet der Besucher zwischen den Straßenschluchten aber auch beschauliche Plätze wie den historischen Botani- schen Garten. Ganz in seiner Nähe, unweit des Hafens, befindet sich die Piazza Marina mit dem Garten der Villa Garibaldi. Im Mittelalter wurde der Platz als Versammlungsort genutzt, Volksfeste fanden hier statt und Gaukler zeigten ihre Künste. Da sich in der Nähe das Inquisitionsgericht befand, fanden auf dem Platz auch Hinrichtungen statt. Die Piazza wird von

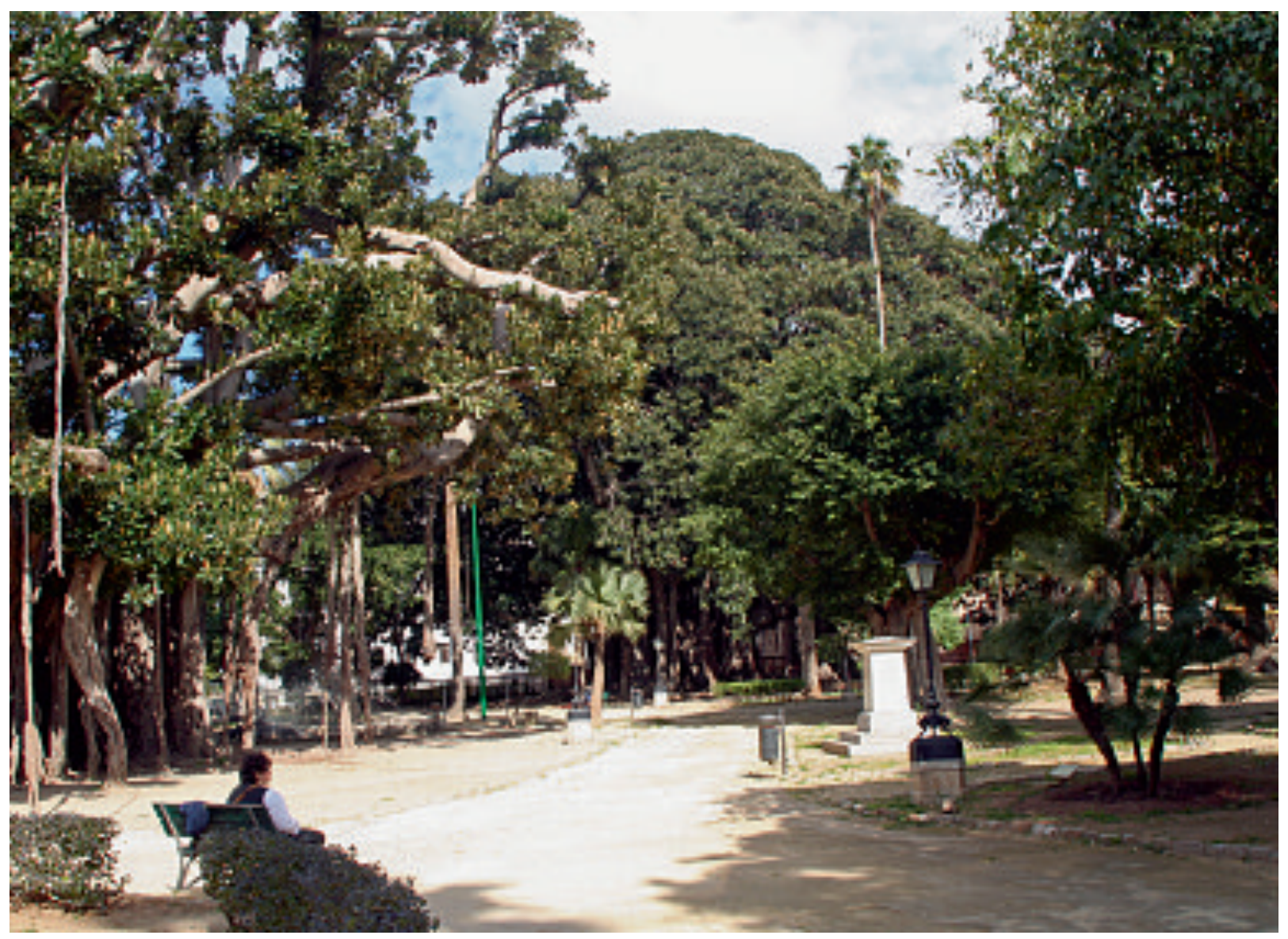


15 Palästen umgeben, darunter der Palazzo Mirto, der besichtigt werden kann.

Heute ist der kleine öffentliche Park für jeden Pflanzenfreund ein Muss. Der nahezu quadratische, etwa 1 ha große Park wurde 1863 von Giovanni Battista Filippo Basile entworfen. Einige alte Bäume, die damals bereits auf der Piazza Marina standen, wurden in die neue Parkbepflanzung integriert. Der Eintritt in den Garten ist kostenlos, seitlich des Eingangs existiert aber ein altes (unbesetztes) Pförtnerhäuschen mit hübschen Holzschnitzereien an der Tür. Im Garten gibt es einige Skulpturen, darunter die 1861 errichtete Büste von Giuseppe Garibaldi (1807-1882), Nationalheld der italienischen Einigungsbewegung, der dem Garten und der Villa seinen Namen gegeben hat.

Umgeben ist der Park von einem schmiedeeisernen Zaun. Wegenetz und Beete haben sich seit der Entstehung kaum verändert. Die Wege führen zur Mitte des Gartens, wo sich ein Brunnen befindet. Beachtenswert ist der alte Baumbestand, wobei noch einige Exemplare mindestens aus der Zeit der Parkgründung stammen und dementsprechend über 150 Jahre alt sind. Allerdings ist die Vielfalt der Gehölze im Garten im Laufe der Jahre durch Überalterung des Bestandes und schlechte Wasserversorgung zurückgegangen.

Im Garten gedeihen viele Palmen wie z. B. Zwergpalme (Chamaerops humilis), Kentiapalme (Howea forsteriana), Sabalpalme (Sabal palmetto), Geleepalme (Butia capitata) oder Livistonie (Livistona chinensis). Die Sizilianer sind stolz auf ihre Zwergpalme, die wild an verschiedenen Stellen der Insel küstennah vorkommt. Oft werden die Palmen von Rindern und Ziegen stark befressen, so dass sie buschig bleiben. Wo sie vor Beweidung geschützt sind wie z. B. an der Nordküste im Schutzgebiet Zingaro, können sie sich zu stattlichen Palmen bis $6 \mathrm{~m}$ Höhe entwickeln. Sie bilden einen Schopf aus

Abb. 1 (Seite 132): Blick auf den größten Feigenbaum. Eine Palme durchdringt seine Krone.

Abb. 2: Eingangshäuschen.

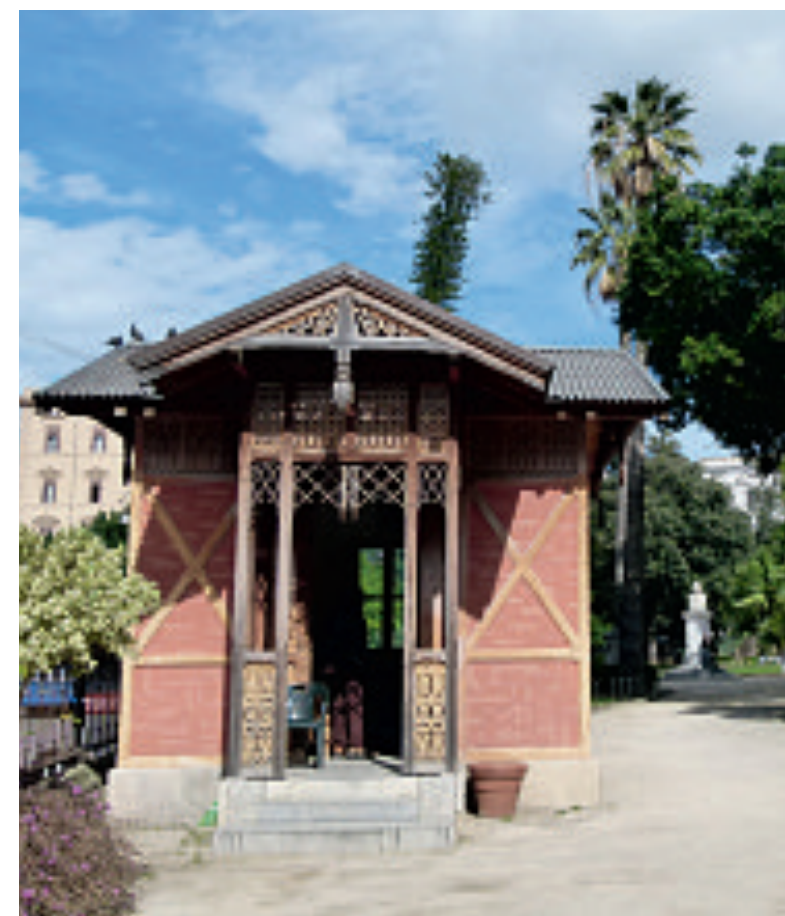

bis $80 \mathrm{~cm}$ breiten Fächerblättern. Die Geleepalme ist relativ frostunempfindlich und soll kurzfristig auch Fröste bis etwa $-10^{\circ} \mathrm{C}$ ertragen. Sie trägt reichlich saftige Früchte, aus denen in Südamerika Gelee, Marmelade oder auch Fruchtwein hergestellt wird. Ein alter Kanarischer Drachenbaum (Dracaena drago) in der Parkmitte zeigt typischen Wuchs mit schirmförmiger Krone und gabeliger Verzweigung. In der Natur ist die Art zwar sehr selten, wird aber häufig als Zierpflanze kultiviert. Aus kleinen weißlichen Blüten entstehen orangebraune Beerenfrüchte.

Die meisten Gehölze im Park sind immergrün, üppig bunte Blumenrabatten fehlen. Die Bäume stammen aus verschiedenen tropischen und subtropischen Gebieten der Erde, von der südamerikanischen Jacaranda (Jacaranda mimosaefolia) über eine asiatische Steineibe (Podocarpus nereifolius) bis hin zur aus Australien stammenden Casuarine (Casuarina tortuosa). Aber auch einige mediterrane Gehölze wie Steineiche (Quercus ilex) und Johannisbrotbaum (Ceratonia siliqua) wurden für die Parkgestaltung verwendet.

Immer wieder faszinierend ist es, in Gärten warmer Regionen auf solche Pflanzen zu treffen, die von zu Hause meist nur als relativ klei- 


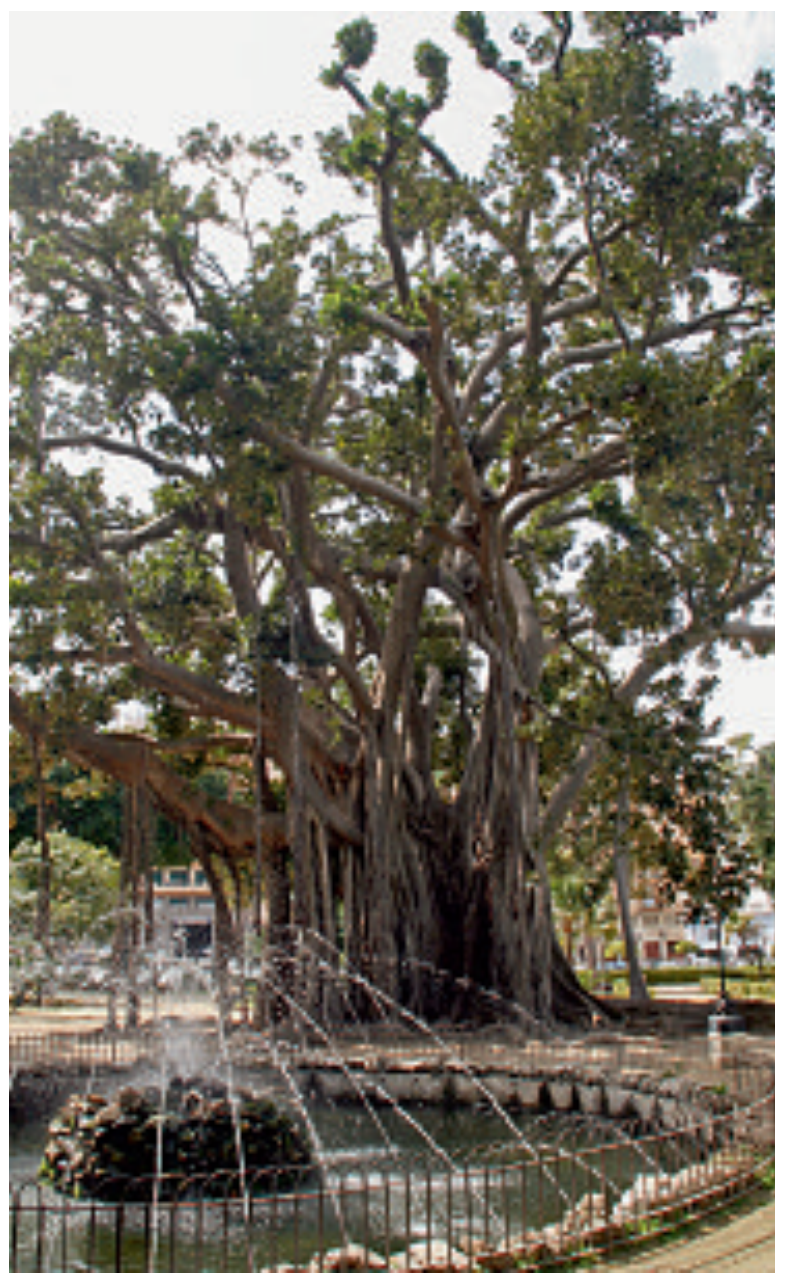

ne Kübelpflanzen bekannt sind. Im Park fällt eine üppige Zimmerlinde (Sparrmannia africana) auf. Sie ist in der Kapregion Südafrikas heimisch und wird dort bis $7 \mathrm{~m}$ hoch. Ihre gelbroten, reizbaren Staubfäden kontrastieren mit den weißen Blütenblättern. Farbakzente unter den Bäumen setzt auch ein strauchförmiges Greiskraut (Senecio petasitis) aus Mexiko. Zahlreiche gelbe Blütenköpfchen sind in kugeligen Ständen vereint. Attraktiv sind zudem die großen Blätter, die in ihrer Form an Blätter von Pelargonien erinnern, weshalb die Art gelegentlich auch als Mexikanische bzw. Kalifornische Geranie bezeichnet wird. Mit $31 \mathrm{~m}$ Höhe ist eine schlanke Norfolk-Tanne (Araucaria heterophylla), bei uns besser bekannt als Zimmertanne, der höchste Baum im Park.

\section{Giganten im Park}

Auf einem Gartenplan sind die 40 bemerkenswertesten Gehölze des Parks markiert. Am be-


eindruckendsten sind drei riesige Feigenbäume (Ficus macrophylla subsp. columnaris), die vor rund 170 Jahren gepflanzt wurden und mehrere etwas kleinere Exemplare von Ficus microcarpa. Zwei der drei großen Feigenbäume haben z. Zt. eine vermutlich aus Sicherheits- und Stabilitätsgründen stark gestutzte Krone, während der dritte das ganze Ausmaß seiner Krone erkennen lässt. In den meisten Gärten, in denen ebenfalls große Feigenbäume wachsen wie z. B. auf Teneriffa, sind die Bäume eingezäunt, damit der Boden um den Baum durch die vielen Besucher

Abb. 3 (links): Brunnen in der Gartenmitte mit einem der gestutzten Feigenbäume.

Abb. 4 (rechts oben): Eine an einen Krakenarm erinnernde Wurzel.

Abb. 5 (rechts unten): Ausschnitt aus dem gewaltigen Wurzelsystem.

Abb. 6 (Seite 135): Bizarres Wurzelgeflecht. 


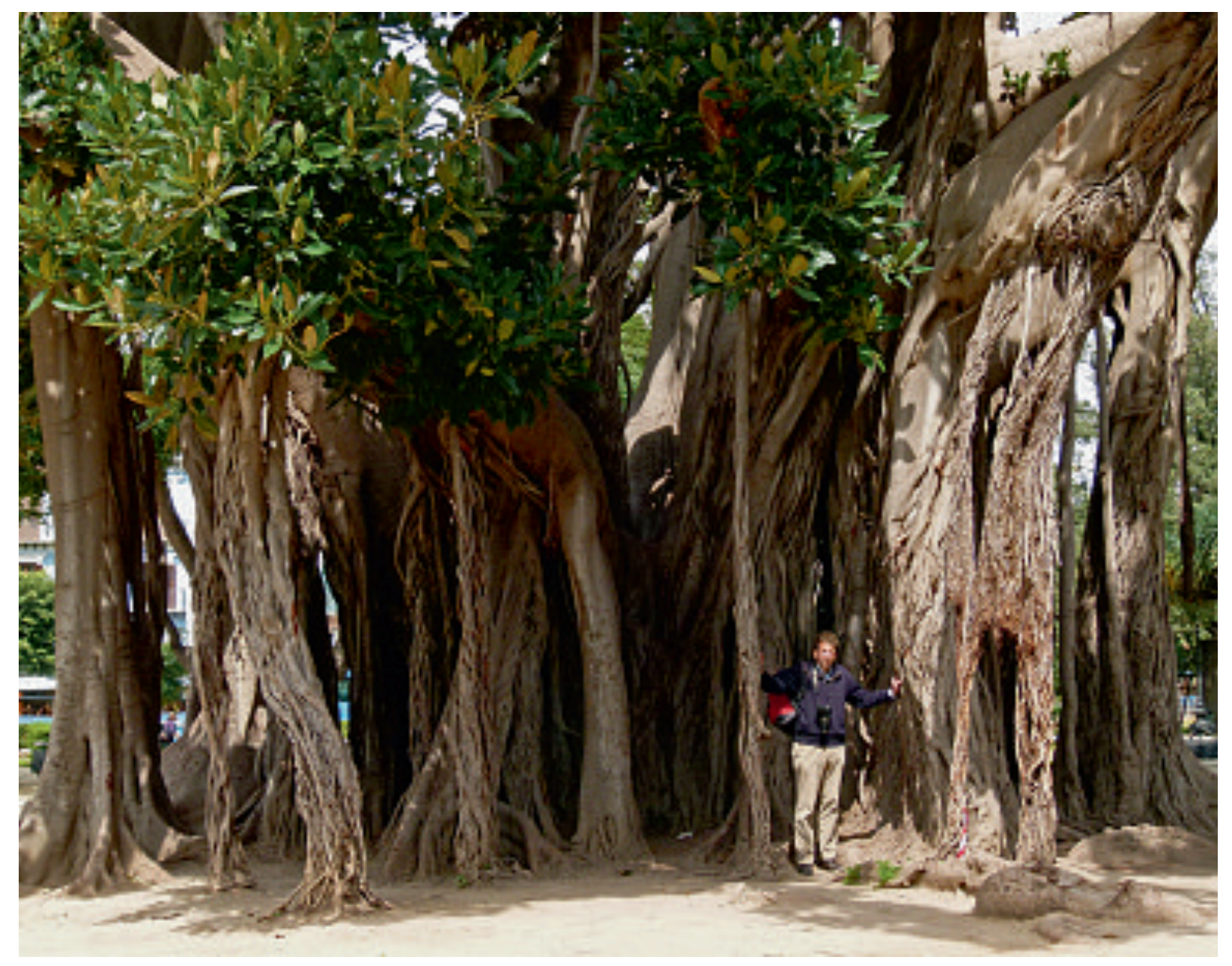

nicht verdichtet wird und die Wurzeln nicht beschädigt werden. Hier in Palermo können die Bäume hautnah und ohne Absperrung erkundet werden, ein ganz besonderes Erlebnis für alle Pflanzenfreunde.

Alle drei Bäume beeindrucken durch ein gigantisches Geflecht ihrer Luftwurzeln, wie es für Würgefeigen charakteristisch ist. Die Wurzeln verwachsen miteinander, verankern sich im Boden und geben dem Baum zusätzlichen Halt. Es entsteht der Eindruck einer Tropfsteinhöhle mit zahlreichen überarmdicken kräftigen Stalaktiten. Die am Boden kriechenden, mitunter pockigen Wurzeln sind Armen von Riesenkraken nicht unähnlich. Der größte Baum nimmt mit seinen Luftwurzeln eine Fläche ein, die schätzungsweise einen Durchmesser von rund $30 \mathrm{~m}$ hat. Geradezu zierlich wirken daneben die Palmen, die sich regelrecht durch die Krone der Feige „durchgearbeitet" haben, vor allem eine schlanke Phoenix canariensis.

\section{Ficus macrophylla}

Die Großblättrige Feige oder auch Moreton Bay Fig Tree (Ficus macrophylla) stammt ursprünglich aus dem Osten Australiens, wird aber wegen ihres bizarren Wuchses häufig in vielen warmen Gebieten als Zierpflanze kultiviert, so z. B. in Neuseeland oder auf Hawaii. Gelegentlich wird sie ebenso wie andere Würgefeigen auch Banyan Fig genannt, obwohl sich dieser Name ursprünglich nur auf die in Indien heimische Art Ficus benghalensis bezog. Es werden zwei Formen von Ficus macrophylla unterschieden. Während die Form macrophylla auf dem australischen Festland vorkommt, ist die Form columnaris auf der Lord Howe Island heimisch. Letztere bildet besonders schöne, säulenförmige Luftwurzeln aus, worauf der Formen-Name Bezug nimmt.

Nach Europa wurde F. macrophylla im 19. Jh. gebracht. In Palermo wurde die Großblättrige Feige erstmals 1840 gepflanzt, darunter auf 

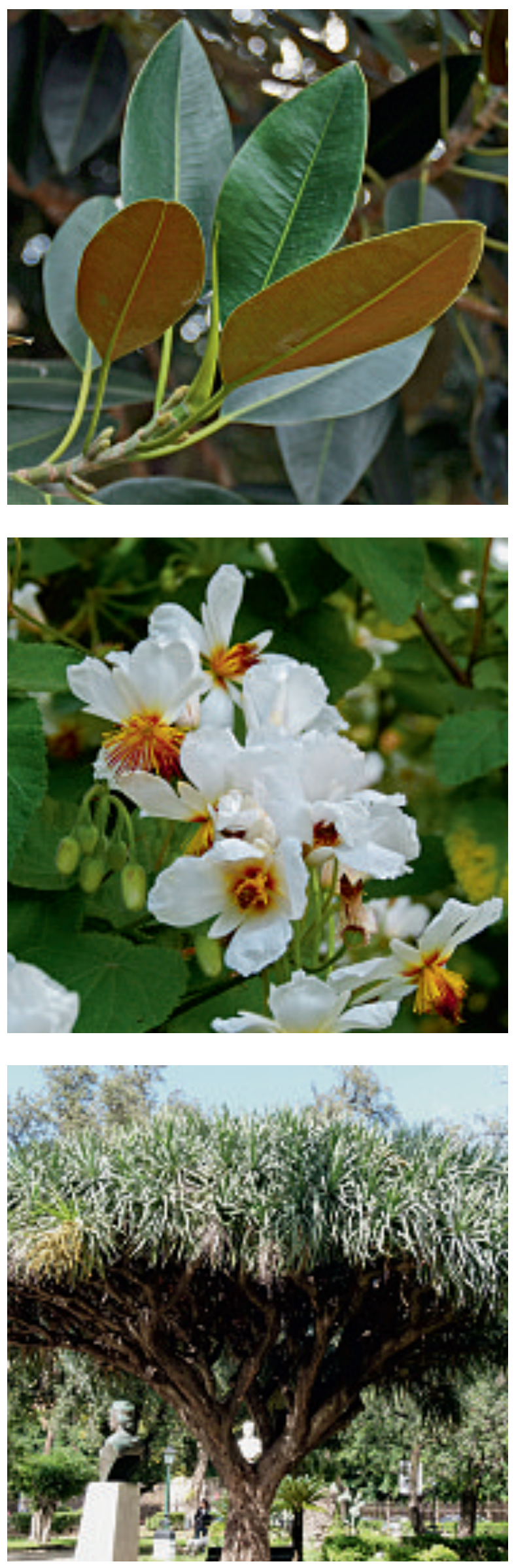

dem Gelände des heutigen Giardino Garibaldi (s. o.) und im Botanischen Garten (Orto Botanico). Der Direktor des Botanischen Gartens, Antonino Borui, beschrieb erst 1897 diese Feigen-Art, wobei sie zunächst Ficus magnolioides wegen ihrer magnolienförmigen ledrigen, bis $25 \mathrm{~cm}$ langen Blätter genannt wurde.

Ficus macrophylla ist eine Würgefeige mit beeindruckendem Wuchs. Sie beginnt ihr Leben meist als Aufsitzerpflanze auf einem anderen Baum, bildet zahlreiche Luftwurzeln, die allmählich den Trägerbaum erwürgen und dem Feigenbaum später Kontakt zum Boden und erhöhte Standfestigkeit verleihen. Ferdinand Mueller (1825-1896), deutsch-australischer Botaniker und Geograph, bezeichnete die Großblättrige Feige als den größten und vermutlich auch beeindruckendsten der australischen Alleebäume. In der Natur kann diese Ficus-Art etwa $60 \mathrm{~m}$ hoch werden und im Extremfall mit ihren Wurzeln eine Fläche von 1 ha einnehmen. Baumschulen warnen deshalb davor, den Baum in kleineren Privatgärten oder zu nah am Haus zu pflanzen.

Die Früchte sind ähnlich gebaut wie normale Essfeigen (Ficus carica), mit einem Durchmesser von etwa $2,5 \mathrm{~cm}$ allerdings kleiner. Die Art lässt sich über Samen oder Stecklinge gut vermehren. Als Zimmer- oder Kübelpflanze wird sie bei uns nicht angeboten, vermutlich kann man ihr nicht ausreichend große Pflanzgefäße bieten.

\section{Dank}

Besonderer Dank gilt Vanessa Pes Lopez und Pamela Passano für kritische Durchsicht des Manuskriptes und Übersetzungen aus dem Italienischen.

Abb. 7 (oben): Die Blätter erinnern an diejenigen von Magnolia grandiflora.

Abb. 8 (Mitte): Die Blüten der Zimmerlinde bringen Farbe in den Park.

Abb. 9 (unten): Ein älterer Drachenbaum im Park. 